\title{
SHROOM3 is a novel component of the planar cell polarity pathway whose disruption causes congenital heart disease
}

Alison Schmidt ${ }^{1}$, Matthew Durbin MS MD², James O'Kane MS $^{2}$, Stephanie M. Ware MD $\mathrm{PHD}^{2}$

${ }^{1}$ Indiana University School of Medicine, Indianapolis, IN, ${ }^{2}$ Department of Pediatrics, Herman B Wells Center for Pediatric Research, Indiana University School of Medicine, Indianapolis, IN

Congenital heart disease (CHD) is the most common cause of death due to birth defects. Despite CHD frequency, the etiology remains mostly unknown. Understanding CHD genetics and elucidating disease mechanism will help establish prognosis, identify comorbidity risks, and develop targeted therapies. CHD often results from disrupted cytoarchitecture and signaling pathways. We have identified a novel CHD candidate SHROOM3, a protein associated with the actin cytoskeleton and the Wnt/Planar Cell Polarity (PCP) signaling pathway. SHROOM3 induces actomyosin constriction within the apical side of cells and is implicated in neural tube defects and chronic renal failure in humans. A recent study demonstrated that SHROOM3 interacts with Dishevelled2 (DVL2), a component of the PCP signaling pathway, suggesting that SHROOM3 serves as an important link between acto-myosin constriction and PCP signaling. PCP signaling establishes cell polarity required for multiple developmental processes, and is required for cardiac development. In Preliminary data we utilized a Shroom 3 gene-trap mouse (Shroom $3^{\text {gtgt }}$ ) to demonstrated that SHROOM3 disruption leads to cardiac defects phenocopy $P C P$ disruption. We also demonstrate that patients with CHD phenotypes have rare and potentially damaging SHROOM3 variants within SHROOM3's PCP-binding domain. We hypothesize SHROOM3 is a novel terminal effector of PCP signaling, and disruption is a novel contributor to CHD. To test this, we assessed genetic interaction between SHROOM3 and PCP during cardiac development and the ultimate effect on cell structure and movement. Heterozygous Shroom $3^{t / g t}$ mice and heterozygous $\mathrm{Dv} / 2^{+/-}$mice are phenotypically normal. We demonstrated genetic interaction between SHROOM3 and PCP signaling by generating compound heterozygous Shroom $3^{\text {togt }} ; D v / 2^{+/}$mice and identifying a Double Outlet Right Ventricle and Ventricular Septal Defect in one embryo. We also observed fewer compound heterozygous mice than anticipated by Mendelian rations (observed: $18.4 \%$; expected: $25 \%$; $n=76$ ), suggesting potential lethality in utero. Immunohistochemistry demonstrates disrupted actomyosin in the SHROOM3 ${ }^{\text {gt/gt }}$ mice, characteristic of PCP disruption. These data help strengthen SHROOM3 as a novel CHD candidate gene and a component of the PCP Signaling pathway. Further characterization of this gene is important for CHD diagnosis and therapeutic development. 